\title{
An Open-loop Calculation in a Path Integral Representation of Affine Nonlinear Optimal Control Systems with Control Costs Quadratic in Control Variables
}

\author{
Teturo ITAMI ${ }^{*}$
}

\begin{abstract}
Monte Carlo methods are applied to an open-loop calculation of affine nonlinear optimal control systems. Nonlinear optimal control systems are represented as an infinite sum or an integration of possible paths connecting initial and final points in their linear wave theories. Weight of each path is a trigonometric function of "action" integral along the path nondimensionalized by a characteristic control constant $H_{R}$ representing strength of fluctuations due to wave action. When this constant is set as a pure imaginary value, $H_{R}=i \tilde{H}_{R}$, trigonometric functions are transformed into exponential functions. We can then consider $\tilde{H}_{R}$ as "temperature" of Boltzmann distributions. The wave function is regarded as a statistical mean under the Boltzmann distribution. Optimal path satisfying conditions at two boundary points is given as a stationary phase of the wave function when the temperature $\tilde{H}_{R}$ is low. Simulation studies for a system with 1-input and 2-states are given.
\end{abstract}

Key Words: two point boundary value problems, nonlinear optimal control, Monte Carlo methods, Boltzmann distribution, quantum mechanical superposition-principle

\section{Introduction}

How to calculate two point boundary value problems is a key task in nonlinear optimal control. Difficulties in finding the answer to such problems lie in the fact that presumed solutions starting with the initial time must be carefully arranged to meet conditions at the final time ${ }^{1)}$. A simple time marching cannot be appllied. While in trying to calculate nonlinear optimal control in feedback form, "superposition-principle" has been introduced by the author ${ }^{2)}$. In this scheme, nonlinearity has been dissolved into wave that associates to the optimal path in state-space. A governing equation has become a linear wave equation with time derivative of the first order. This equation has been set up as the final value problem. Paths in state-space are collected to generate such wave. This is the path integral representation of nonlinear control. We can then transform two point boundary value problems of ordinary differential equations into final value problems of the time first order partial differential wave equations.

We propose in this paper a method to calculate two point boundary value problems of affine nonlinear control systems in a path integration scheme. Each path in such an integral has weight $\exp \left(\frac{i}{H_{R}} S_{\pi}\right)$ of exponential function of its action $S_{\pi}$ along the path $\pi$ nondimension-

\footnotetext{
* Kure Research Laboratory, Babcock-Hitachi Co.Ltd. (Received September 13, 2002)

(Revised November 12, 2003)
}

alized by a new designer's constant $H_{R}$. After transforming $H_{R}$ to a pure imaginary number, $H_{R}=i \tilde{H}_{R}{ }^{3)}$, when we regard this real constant $\tilde{H}_{R}$ as "temperature", the weight $\exp \left(\frac{1}{\tilde{H}_{R}} S_{\pi}\right)$ can be regarded as the Boltzmann factor. The wave function represented by path integral can be thought of as a statistical mean value under the Boltzmann distribution. Because in the limit $H_{R} \rightarrow 0$ the stationary phase of a path integral form of the wave function represents the optimal path, we attain an algorithm to calculate a path satisfying two point boundary values using a Boltzmann distribution at "low temperature". We can then make fully use of various methods to calculate statistical properties. Among possible ways, we take here methods of Monte Carlo integration ${ }^{4)}{ }^{5)}$. Recently there appears an idea ${ }^{6)}$ of introducing probability or stochasticity into deterministic control theories. Present report can propose another concept regarding probabilistic theoy of deterministic nonlinear control systems. Moreover our theory stands on a firm theoretical foundation of the established tool of Monte Carlo integrations.

The paper is organized as follows. In Section 2 we take nonlinear optimal control systems given by Eqs.(1) and (2) below, which are not necessarily of affine type. After a brief review of "superposition-principle", a wave function is written down as a path integral over the state variable and the canonical momentum. This is used to calculate optimal path or problem with two boundary conditions at initial and final points. For affine systems with control 
specifications described by functions quadratic in control variables, the integrand of the path integral is shown in Section 3 to be quadratic in the canonical momentum. To reduce a calculational burden, the path integration with respect to the canonical momentum can be done analytically. After transforming the designer's constant to a pure imaginary number, $H_{R}=i \tilde{H}_{R}$, we attain the wave function for affine systems as a statistical mean under the Boltzmann distribution. This statistical mean is subsequently calculated by Monte Carlo algorithm. An explanation of our algorithm is posed using a simple system with 1 -input and 2-state variables, which will be easily extended to general systems with $m$-input and $n$-state variables. Section 4 is devoted to simulation studies on a typical nonlinear system with 1-input and 2-states. In this example, the non-quadratic functions of the terminal cost are chosen. Errors of the canonical equations of motion are examined for various values of the designer's constant $H_{R}$. Summary and discussion are given in Section 5. In Appendix A, a "c-number Hamiltonian" is introduced as an extension of the Hamiltonian and is explicitly calculated for affine systems. Using the c-number Hamiltonian, canonical equations of motion and a final condition are derived as a stationary phase condition of the path integral in Appendix B. Finally in Appendix $C$, a relation between the canonical equations of motion of canonical momentum and the stationary phase of the path integral only over the state variables is clarified.

\section{Nonlinear optimal control systems as a stationary phase of path integrals ${ }^{2)}$}

In this section we take general nonlinear optimal control systems which includes affine systems. A generalization of the Schrödinger equation and a path integral representation of a wave function are given.

\subsection{A generalized Schrödinger equation}

A system to be considered is defined by a state equation

$$
\dot{\vec{x}}=\vec{f}(\vec{x}, \vec{u})
$$

with an initial condition at $t=t_{I}, \vec{x}\left(t_{I}\right)=\vec{x}_{I}$ and a control specification.

$$
\delta P I \equiv \delta\left\{\Phi\left(\vec{x}_{F}\right)+\int_{t_{I}}^{t_{F}} d t L(\vec{x}, \vec{u})\right\}=0
$$

A final value $\vec{x}\left(t_{F}\right)=\vec{x}_{F}$ is calculated by Eq.(4) below as a result of the optimality condition. In the above equations (1) and (2), $\vec{x} \equiv\left(x_{1}, x_{2}, \ldots, x_{i}, \ldots, x_{n}\right)^{T}$ is the state variable and $\vec{u} \equiv\left(u_{1}, u_{2}, \ldots, u_{\alpha}, \ldots, u_{m}\right)^{T}$ is the control variable. Using the costate variable $\vec{\lambda} \equiv\left(\lambda_{1}, \lambda_{2}, \ldots, \lambda_{i}, \ldots, \lambda_{n}\right)^{T}$, Eqs.(1) and (2) are summarized as the following mini- mization equation

$$
\delta P I^{\prime} \equiv \delta\left\{\Phi\left(\vec{x}_{F}\right)+\int_{t_{I}}^{t_{F}} d t L^{\prime}(\vec{x}, \vec{u}, \vec{\lambda} ; \dot{\vec{x}})\right\}=0
$$

with a final condition.

$$
\vec{\lambda}\left(t_{F}\right)=\frac{\partial \Phi\left(\vec{x}_{F}\right)}{\partial \vec{x}_{F}}
$$

In Eq.(3) above, we defined an extended Lagrangian

$$
L^{\prime}(\vec{x}, \vec{u}, \vec{\lambda} ; \dot{\vec{x}}) \equiv L(\vec{x}, \vec{u})+\overleftarrow{\lambda} \cdot(\vec{f}(\vec{x}, \vec{u})-\dot{\vec{x}})
$$

with $\overleftarrow{\lambda} \equiv\left(\lambda_{1}, \lambda_{2}, \ldots, \lambda_{i}, \ldots, \lambda_{n}\right)$ (the transposed $\vec{\lambda}$ ).

Regarding this $L^{\prime}$ as a Lagrangian of the system, we can deal with the system in standard canonical dynamics. We have $\vec{p}_{x} \approx-\vec{\lambda}^{(1)}$ as a canonical momentum corresponding to the state variable $\vec{x}$. Under an assumption that the following matrix is regular

$$
b_{\alpha, \beta} \equiv \frac{\partial^{2}(L+\overleftarrow{\lambda} \cdot \vec{f})}{\partial u_{\alpha} \partial u_{\beta}}
$$

the optimality conditions of control are derived as follows.

$$
\frac{\partial(L+\overleftarrow{\lambda} \cdot \vec{f})}{\partial u_{\alpha}}=0
$$

Applying a Legendre transformation to the dynamical system defined by $L^{\prime}$ of Eq.(5) we can calculate a Hamiltonian as follows.

$$
H(\vec{x}, \vec{u}, \vec{\lambda})=-L(\vec{x}, \vec{u})-\overleftarrow{\lambda} \cdot \vec{f}(\vec{x}, \vec{u})
$$

In our scheme of optimizing nonlinear control, the control variable $\vec{u}$ and the costate variable $\vec{\lambda}$ have corresponding operator representations of $\hat{\vec{u}}$ and $\hat{\vec{\lambda}}$, respectively. Substitution of these operators into the Hamiltonian $H$ defined by Eq.(8) leads to a linear operator form of the Hamiltonian, a Hamiltonian operator $\hat{H}=H(\vec{x}, \hat{\vec{u}}, \hat{\vec{\lambda}})$. Applying this Hamiltonian operator, our governing equation for feedback synthesis is the following linear wave equation, what we call a generalized Schrödinger equation.

$$
i H_{R} \frac{\partial \psi\left(\vec{x}, t ; H_{R}\right)}{\partial t}=\hat{H} \psi\left(\vec{x}, t ; H_{R}\right)
$$

with a final condition

$$
\psi\left(\vec{x}, t_{F} ; H_{R}\right)=R_{F}(\vec{x}) e^{-\frac{i}{H_{H}} \Phi(\vec{x})}
$$

where the terminal cost function $\Phi\left(\vec{x}_{F}\right)$ of Eq.(3) is used. When control designers choose the parameter $H_{R}$ representing strength of wave action as real and positive, a real part of the linear wave equation (9) leads ${ }^{2)}$ to a generalization of nonlinear partial differential equation of Hamilton and Jacobi imposed on a function $S^{q}$ given by Eq.(11) below. For the absolute value of the final condition (10), we must choose this $R_{F}(\vec{x})$ as a positive-definite function and having no parametric dependence on $H_{R}$. The wave

(1) The equality denoted by ' $\approx$ ' is a weak one satisfied only on the submanifold of the systems ${ }^{2}$ ) 
function $\psi$ is a complex-valued function of $\vec{x}, t$ defined over $\Omega_{x} \times\left[t_{I}, t_{F}\right]$ and is at least 2-times differentiable, where $\Omega_{x} \subset R^{n}$ is a compact subset of $R^{n}$. We can decompose the complex-valued wave function $\psi \in C$.

$$
\psi\left(\vec{x}, t ; H_{R}\right)=R^{q}\left(\vec{x}, t ; H_{R}\right) e^{\frac{i}{H_{R}} S^{q}\left(\vec{x}, t ; H_{R}\right)}
$$

The phase function $S^{q}$ and the absolute value function $R^{q}$ are defined over $\Omega_{x} \times\left[t_{I}, t_{F}\right]$ and at least 2-times differentiable. These properties are induced from those of the wave function $\psi$.

\subsection{A path integral}

The generalized Schrödinger equation (9) is linear, so we can calculate the wave function at an arbitrary time as a superposition of elementary waves, each of which is a result of propagation of the wave at time $t_{F}$ having a peak $\psi\left(\vec{x}, t_{F} ; H_{R}\right)$ at an arbitrary location $\vec{x}=\vec{x}_{F}$. We can formulate such superposition using a kernel of the partial differential equation (9). The kernel $G\left(\vec{x}, t \leftarrow \vec{x}_{F}, t_{F}\right)$ is defined as a solution to Eq.(9) such that the final condition is the following delta function (13) having a peak at $\vec{x}_{F}$. We have the following equations.

$$
\begin{aligned}
& i H_{R} \frac{\partial}{\partial t} G\left(\vec{x}, t \leftarrow \vec{x}_{F}, t_{F}\right)=\hat{H} G\left(\vec{x}, t \leftarrow \vec{x}_{F}, t_{F}\right) \\
& \left.G\left(\vec{x}, t \leftarrow \vec{x}_{F}, t_{F}\right)\right|_{t=t_{F}}=\delta\left(\vec{x}-\vec{x}_{F}\right)
\end{aligned}
$$

We emphasize by using a notation $\vec{x}, t \leftarrow \vec{x}_{F}, t_{F}$ in the argument of $G$ that an effect of a wave at $\vec{x}_{F}, t_{F}$ propagates to $\vec{x}, t$. This kernel $G$ is calculated as follows

$$
G\left(\vec{x}, t \leftarrow \vec{x}_{F}, t_{F}\right)=e^{-\frac{i}{t_{R}} \hat{H}\left(t-t_{F}\right)} \delta\left(\vec{x}-\vec{x}_{F}\right)
$$

where the partial derivative operators appearing in the Hamiltonian operator $\hat{H}$ act on the variable $\vec{x}$ of the delta function. Because Eq.(9) is linear, the wave function at an arbitrary time $t$ is calculated using the kernel as follows

$$
\psi\left(\vec{x}, t ; H_{R}\right)=\int d \vec{x}_{F} G\left(\vec{x}, t \leftarrow \vec{x}_{F}, t_{F}\right) \psi\left(\vec{x}_{F}, t_{F} ; H_{R}\right)(15)
$$

This represents the Huygens' principle. The kernel $G\left(\vec{x}, t \leftarrow \vec{x}_{F}, t_{F}\right)$ has the property that for $t<\forall t_{a}<t_{F}$

$$
\begin{aligned}
& G\left(\vec{x}, t \leftarrow \vec{x}_{F}, t_{F}\right)= \\
& \quad \int d \vec{x}_{a} G\left(\vec{x}, t \leftarrow \vec{x}_{a}, t_{a}\right) G\left(\vec{x}_{a}, t_{a} \leftarrow \vec{x}_{F}, t_{F}\right)
\end{aligned}
$$

Using this repeatedly, the kernel is expressed as a multiple integral of the kernels.

$$
\begin{gathered}
G\left(\vec{x}, t \leftarrow \vec{x}_{F}, t_{F}\right)=\int \cdots \int \cdots \int d \vec{x}_{1} \cdots \cdots d \vec{x}_{N-1} \\
G\left(\vec{x}, t \leftarrow \vec{x}_{1}, t_{1}\right) G\left(\vec{x}_{1}, t_{1} \leftarrow \vec{x}_{2}, t_{2}\right) \\
\cdots G\left(\vec{x}_{k-1}, t_{k-1} \leftarrow \vec{x}_{k}, t_{k}\right) \cdots \\
G\left(\vec{x}_{N-2}, t_{N-2} \leftarrow \vec{x}_{N-1}, t_{N-1}\right) \\
G\left(\vec{x}_{N-1}, t_{N-1} \leftarrow \vec{x}_{F}, t_{F}\right)
\end{gathered}
$$

where $t_{k}=t+k \Delta t$ and $\Delta t \equiv \frac{t_{F}-t}{N}>0\left(t_{0}=t\right.$ and

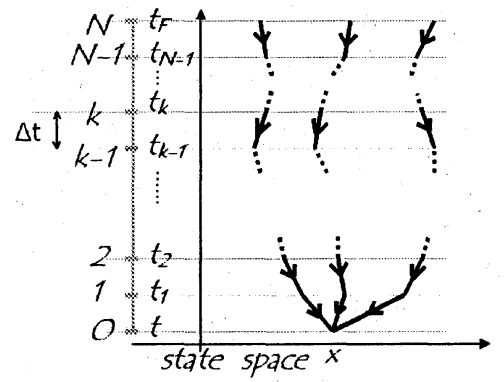

Fig. 1 The Huygens' principle repeatedly applied to infinitesimal time intervals. Arrows correspond to those in Eq.(17).

$\left.t_{N}=t_{F}\right)$ as shown in Fig. 1. From Eq.(14) we can calculate the kernel for the infinitesimal time interval.

$$
\begin{gathered}
G\left(\vec{x}_{k-1}, t_{k-1} \leftarrow \vec{x}_{k}, t_{k}\right) \\
=e^{-\frac{i}{H_{R}} \hat{H}\left(t_{k-1}-t_{k}\right)} \delta\left(\vec{x}_{k-1}-\vec{x}_{k}\right) \\
=\left(1-\frac{i}{H_{R}} \hat{H}\left(t_{k-1}-t_{k}\right)\right) \delta\left(\vec{x}_{k-1}-\vec{x}_{k}\right) \\
\quad+O\left(\left|t_{k-1}-t_{k}\right|^{2}\right)
\end{gathered}
$$

where as the partial derivatives in $\hat{H}$ we must set $\frac{\partial}{\partial \vec{x}_{k-1}}$. Although the Hamiltonian operator $\hat{H}$ is never an ordinary number, this $\hat{H}$ can be represented by a superposition of ordinary functions $H^{[\theta]}\left(\vec{p}_{x}, \vec{x} ; H_{R}\right)$ as is shown in Appendix A, Eq.(A.4). This $H^{[\theta]}$ is an extension of the conventional Hamiltonian $H$ in that $H^{[\theta]}$ is a sum of $H$ and effects of $H_{R}$. We can then calculate the second term $\hat{H} \delta\left(\vec{x}_{k-1}-\vec{x}_{k}\right)$ of Eq.(18) to obtain

$$
\begin{aligned}
& \hat{H} \delta\left(\vec{x}_{k-1}-\vec{x}_{k}\right)=\int \frac{d \vec{p}_{x_{k}}}{\left(2 \pi H_{R}\right)^{n}}
\end{aligned}
$$

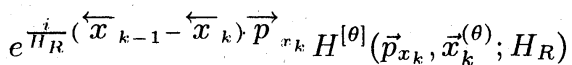

as derived in Appendix A, Eq.(A. 7). We thus have

$$
\begin{array}{r}
G\left(\vec{x}_{k-1}, t_{k-1} \leftarrow \vec{x}_{k}, t_{k}\right)= \\
\int \frac{d \vec{p}_{x_{k}}}{\left(2 \pi H_{R}\right)^{n}} \exp \left(\frac { i } { H _ { R } } \left[\left(\overleftarrow{x}_{k-1}-\overleftarrow{x}_{k}\right) \cdot \vec{p}_{x_{k}}\right.\right. \\
\left.\left.-\left(t_{k-1}-t_{k}\right) H^{[\theta]}\left(\vec{p}_{x_{k}}, \vec{x}_{k}^{(\theta)} ; H_{R}\right)\right]\right) \\
+O\left(\left|t_{k-1}-t_{k}\right|^{2}\right)
\end{array}
$$

In this formula, $\vec{x}_{N}=\vec{x}_{F}, \vec{x}_{0}=\vec{x}$ and $\vec{x}_{k}^{(\theta)} \equiv\left(\frac{1}{2}+\theta\right) \vec{x}_{k}+$ $\left(\frac{1}{2}-\theta\right) \vec{x}_{k-1}$. Substituting this into Eq.(17) and noting that $t_{k-1}-t_{k}=-\Delta t$ and

$$
\begin{aligned}
\left(\overleftarrow{(}_{k-1}\right. & \left.-\overleftarrow{x}_{k}\right) \cdot \vec{p}_{x_{k}} \\
& -\left(t_{k-1}-t_{k}\right) H^{[\theta]}\left(\vec{p}_{x_{k}}, \vec{x}_{k}^{(\theta)} ; H_{R}\right) \\
= & -\Delta t\left\{\frac{\overleftarrow{x}_{k}-\overleftarrow{x}_{k-1}}{\Delta t} \vec{p}_{x_{k}}-H^{[\theta]}\left(\vec{p}_{x_{k}}, \vec{x}_{k}^{(\theta)} ; H_{R}\right)\right\}
\end{aligned}
$$

Eqs.(10) and (15) lead to the wave function at an arbitrary 
time as the following formula of the multiple integral

$$
\begin{gathered}
\psi\left(\vec{x}, t ; H_{R}\right)=\int d \vec{x}_{F} \int d \vec{x}_{1} \cdots \cdots d \vec{x}_{N-1} \\
\int \ldots \int \frac{d \vec{p}_{x_{1}}}{\left(2 \pi H_{R}\right)^{n}} \cdots \frac{d \vec{p}_{x_{k}}}{\left(2 \pi H_{R}\right)^{n}} \cdots \frac{d \vec{p}_{x_{N}}}{\left(2 \pi H_{R}\right)^{n}} \\
\exp \left(-\frac{i}{H_{R}} \Delta t\left\{\left[\frac{\overleftarrow{x}_{1}-\overleftarrow{x}}{\Delta t} \vec{p}_{x_{1}}\right.\right.\right. \\
\left.-H^{[\theta]}\left(\vec{p}_{x_{1}}, \vec{x}_{1}^{(\theta)} ; H_{R}\right)\right]+\cdots \\
+\frac{i}{H_{R}} \Delta t\left[\frac{\overleftarrow{x}_{F}-\overleftarrow{x}_{N-1}}{\Delta t} \vec{p}_{x_{N}}-H^{[\theta]}\left(\vec{p}_{x_{N}}, \vec{x}_{N}^{(\theta)} ; H_{R}\right)\right] \\
\left.\left.+\frac{1}{\Delta t} \Phi\left(\vec{x}_{F}\right)\right\}\right) R_{F}\left(\vec{x}_{F}\right) \\
+O\left(\left|t_{1}-t_{0}\right|^{2}\right)+\cdots+O\left(\left|t_{N}-t_{N-1}\right|^{2}\right)
\end{gathered}
$$

In $\Delta t \rightarrow 0$, a stationary phase of the integral is shown in Appendix B to lead to the final condition (4) and a set of ordinary differential equations of the state variable $\vec{x}$ and the canonical momenum $\vec{p}_{x}$ if $H^{[\theta]}$ has no singularity at $H_{R}=0$. When a condition $\left.H^{[\theta]}\right|_{H_{R}=0}=H$ is furthermore satisfied, these ordinary differential equations are nothing but the canonical equations of motion. Setting $H_{R}$ as a general complex number, we take a limit $H_{R} \rightarrow 0$ under an assumption that we attain the same result in every direction of $H_{R} \rightarrow 0$ on the complex plane. This assumption must be examined in forthcoming reports.

\section{Algorithm for affine systems}

When we set $t=t_{I}$ and $\vec{x}=\vec{x}_{I}$, our starting formula to calculate two point boundary value problems is Eq.(22). In this section, an algorithm of calculating the optimal path for a system defined ${ }^{(2)}$ by the following equations.

$$
\begin{aligned}
& f_{i}(\vec{x}, \vec{u})=g_{i, \underline{\alpha}}(\vec{x}) u_{\underline{\alpha}}+F_{i}(\vec{x}) \\
& L(\vec{x}, \vec{u})=u_{\underline{\alpha}} R_{\underline{\alpha}, \underline{\beta}} u_{\underline{\beta}}+V_{\text {cost }}(\vec{x})
\end{aligned}
$$

is proposed, where $g_{i, \alpha}, F_{i}$ and $V_{\text {cost }}$ are at least 1-time differentiable. The system defined by Eq.(23) is an affine system, while Eq.(24) states that an optimality of the system is determined by a function $L$ quadratic in control variables $\vec{u}$. We, simply call the system defined by Eqs.(23) and (24) the affine system in brief throughout the paper. As is summarized in Appendix A, for affine systems defined by Eqs.(23) and (24) operators $\hat{\vec{u}}$ and $\hat{\vec{\lambda}}$ are explicitly given by Eqs.(A.8) and (A.9), respectively. The Hamiltonian operator $\hat{H}$ is given also explicitly by Eq.(A. 10). As is shown also in Appendix A, the c-number Hamiltonian $H^{[\theta]}$ is calculated as a quadratic form in the

(2) Underlines are understood as the summation over the repeated and underlined suffixes, $A_{\underline{i}} B_{\underline{i}}=\sum_{i=1}^{n} A_{i} B_{i}$ and $C_{\underline{\alpha}} D_{\underline{\alpha}}=\sum_{\alpha=1}^{m} C_{\alpha} D_{\alpha}$. canonical momentum $\vec{p}_{x}$, with no singularity at $H_{R}=0$ and $\left.H^{[\theta]}\right|_{H_{R}=0}=H$. We first analytically carry out an integration (22) over $\vec{p}_{x}$ to reduce a calculational burden. The canonical equation of motion of the canonical momentum $\vec{p}_{x}$ is shown in Appendix $\mathrm{C}$ to be obtained also as the stationary phase condition of the integral only with respect to the state variable $\vec{x}$. When this path integral only over the state variable has a similarity with an established formula, we can fully utilize methods calculating the formula in evaluating our path integral. Let us temporarily try to transform the designer's constant $H_{R}$ to a pure imaginary number, $H_{R}=i \tilde{H}_{R}$ with real $\tilde{H}_{R}$. A ratio of the transformed constant $\tilde{H}_{R}$ to the time difference $\Delta t, T=\frac{\tilde{H}_{R}}{\Delta t}$, is then identified with a "temperature" $T$. We thus attain the wave function (22) as an established formula of a statistical mean under the Boltzmann distribution at temperature $T$. Because the optimal path is extracted in the limit $H_{R} \rightarrow 0$ as the stationary phase of Eq.(22), we can set forth an algorithm of calculating the optimal path in the corresponding limit of "low temperature" $T=\frac{\tilde{H}_{R}}{\Delta t} \rightarrow 0$.

For simplicity of explanation, we take a typical affine system with 1 -input and 2-state variables.

$$
\begin{aligned}
& \dot{x}_{1}=F_{1}(\vec{x}) \\
& \dot{x}_{2}=g_{2}(\vec{x}) u+F_{2}(\vec{x})
\end{aligned}
$$

and with the control specification.

$$
L=\frac{m}{2} u^{2}+V_{\text {cost }}(\vec{x})
$$

Calculations of this section are also easily extended to general systems with $m$-control and $n$-state variables, as will be reported elsewhere. The conventional Hamiltonian (8) is calculated as follows.

$$
H=\frac{g_{2}(\vec{x})^{2}}{2 m} p_{x, 2}^{2}-V_{\text {cost }}(\vec{x})+F_{1}(\vec{x}) p_{x, 1}+F_{2}(\vec{x}) p_{x, 2}(28)
$$

We must replace this Hamiltonian $H$ with the c-number Hamiltonian $H^{[\theta]}$ to treat superposition principle of nonlinear control. This is easily done by replacing the conventional function $V_{\text {cost }}, F_{1}$ and $F_{2}$ with new functions, defined by Eqs.(A. 15) and (A. 16), $\mathcal{V}_{\text {cost }}, \mathcal{F}_{1}$ and $\mathcal{F}_{2}$, respectively, which are dependent both on $H_{R}$ and $\theta$. The c-number Hamiltonian is then given.

$$
\begin{aligned}
H^{[\theta]}\left(\vec{p}_{x}, \vec{x} ; H_{R}\right)=\frac{g_{2}(\vec{x})^{2}}{2 m} p_{x, 2}^{2}-\mathcal{V}_{\text {cost }}\left(\vec{x} ; H_{R} ; \theta\right) \\
\quad+\mathcal{F}_{1}\left(\vec{x} ; H_{R} ; \theta\right) p_{x, 1}+\mathcal{F}_{2}\left(\vec{x} ; H_{R} ; \theta\right) p_{x, 2}
\end{aligned}
$$

\subsection{The wave function as a statistical mean} under Boltzmann distributions

We first integrate over the canonical momentum variables $\vec{p}_{x_{k}}$. Familiar integration formulas are used 


$$
\begin{aligned}
& \int_{-\infty}^{\infty} d p e^{i a p}=2 \pi \delta(a) \\
& \int_{-\infty}^{\infty} d p e^{a p^{2}+b p}=\sqrt{\frac{\pi}{-a}} e^{-\frac{b^{2}}{4 a}}
\end{aligned}
$$

to have the general $k$-th term $(k=1,2, . ., N)$ of Eq.(22).

$$
\begin{gathered}
\iint \frac{d p_{x_{k}, 1} d p_{x_{k}, 2}}{\left(2 \pi H_{R}\right)^{2}} \exp \left(-\frac{i}{H_{R}} \Delta t\left[\frac{\overleftarrow{x}_{k}-\overleftarrow{x}_{k-1}}{\Delta t} \vec{p}_{x k}\right.\right. \\
\left.\left.-H^{[\theta]}\left(\vec{p}_{x k}, \vec{x}_{k}^{(\theta)} ; H_{R}\right)\right]\right) \\
=\frac{1}{\left(2 \pi H_{R}\right)^{2}} \frac{2 \pi H_{R}}{-\Delta t} \delta\left(\frac{x_{k, 1}-x_{k-1,1}}{\Delta t}-\mathcal{F}_{1}\left(\vec{x}_{k}^{(\theta)} ; H_{R} ; \theta\right)\right) \\
\sqrt{\frac{2 m \pi H_{R}}{-i \Delta t}} \sqrt{\frac{1}{\left(g_{2}\left(\vec{x}_{k}^{(\theta)}\right)\right)^{2}}} \exp \left(-\frac{i}{H_{R}} \Delta t\right. \\
{\left[\frac{m}{2\left(g_{2}\left(\vec{x}_{k}^{(\theta)}\right)\right)^{2}}\left(\frac{x_{k, 2}-x_{k-1,2}}{\Delta t}-\mathcal{F}_{2}\left(\vec{x}_{k}^{(\theta)} ; H_{R} ; \theta\right)\right)^{2}\right.} \\
\left.\left.+\mathcal{V}_{\text {cost }}\left(\vec{x}_{k}^{(\theta)} ; H_{R} ; \theta\right)\right]\right)
\end{gathered}
$$

We thus obtain the following formula.

$$
\begin{aligned}
\psi\left(\vec{x}, t ; H_{R}\right)= & \int d \vec{x}_{1} \int d \vec{x}_{2} \cdots \int d \vec{x}_{N} f\left(\vec{x} ; \vec{x}_{1}, \ldots, \vec{x}_{N} ; H_{R} ; \theta\right) \\
& \exp \left(-\frac{i}{H_{R}} \Delta t E\left(\vec{x} ; \vec{x}_{1}, \ldots, \vec{x}_{N} ; H_{R} ; \theta\right)\right)
\end{aligned}
$$

where

$$
\begin{aligned}
& f\left(\vec{x} ; \vec{x}_{1}, \ldots, \vec{x}_{N} ; H_{R} ; \theta\right)= \\
& A R_{F}\left(\vec{x}_{N}\right) \prod_{k=1}^{N} \sqrt{\frac{1}{\left(g_{2}\left(\vec{x}_{k}^{(\theta)}\right)\right)^{2}}} \\
& \quad \delta\left(\frac{x_{k, 1}-x_{k-1,1}}{\Delta t}-\mathcal{F}_{1}\left(\vec{x}_{k}^{(\theta)} ; H_{R} ; \theta\right)\right)
\end{aligned}
$$

with a trivial constant, $A \equiv\left(\frac{1}{\left(2 \pi H_{R}\right)^{2}} \frac{2 \pi H_{R}}{-\Delta t} \sqrt{\frac{2 m \pi H_{R}}{-i \Delta t}}\right)^{N}$ and

$$
\begin{aligned}
& E\left(\vec{x} ; \vec{x}_{1}, \ldots, \vec{x}_{N} ; H_{R} ; \theta\right)= \\
& \begin{aligned}
\sum_{k=1}^{N}\left[\frac{m}{2\left(g_{2}\left(\vec{x}_{k}^{(\theta)}\right)\right)^{2}}\left(\frac{x_{k, 2}-x_{k-1,2}}{\Delta t}-\mathcal{F}_{2}\left(\vec{x}_{k}^{(\theta)} ; H_{R} ; \theta\right)\right)^{2}\right. \\
\left.+\mathcal{V}_{\text {cost }}\left(\vec{x}_{k}^{(\theta)} ; H_{R} ; \theta\right)\right]+\frac{\Phi\left(\vec{x}_{N}\right)}{\Delta t}
\end{aligned}
\end{aligned}
$$

As is shown in Appendix A that for $H_{R}=0$ we have $\mathcal{V}_{\text {cost }}=V_{\text {cost }}, \mathcal{F}_{1}=F_{1}$ and $\mathcal{F}_{2}=F_{2}$. It is then possible to "interprete" the above Eqs.(34) and (35) in the following way. The delta function with $H_{R}=0$ in Eq.(34) expresses the state equation (25) . The first term of the function $E$, Eq.(35), multiplied by $\Delta t$ has a form of the integration of the function $L$, control specification (27), along the path. In this expression, control variable $u$ is solved out using the state equation (26), after replacements of $V_{\text {cost }}, F_{1}$ and $F_{2}$ with $\mathcal{V}_{\text {cost }}, \mathcal{F}_{1}$ and $\mathcal{F}_{2}$, respectively. This formula $\Delta t E$ also contains the terminal cost function $\Phi$.

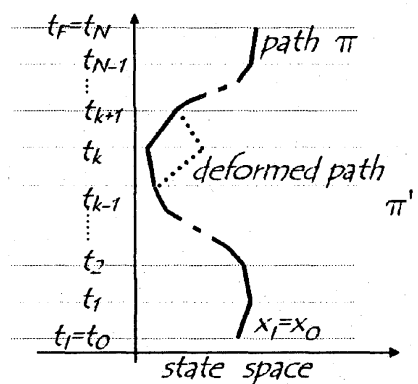

Fig. 2 A path $\pi$ and a deformed path $\pi^{\prime}$

After these calculations, the new designer's constant is set as a pure imaginary number, $H_{R}=i \tilde{H}_{R}$. The constant $\tilde{H}_{R}$ is real. We then arrive at the following formula.

$$
\begin{aligned}
\psi\left(\vec{x}, t ; i \tilde{H}_{R}\right)= & \int d \vec{x}_{1} \int d \vec{x}_{2} \cdots d \vec{x}_{N} f\left(\vec{x} ; \vec{x}_{1}, \ldots, \vec{x}_{N} ; i \tilde{H}_{R} ; \theta\right) \\
& \exp \left(-\frac{1}{\tilde{H}_{R}} \Delta t E\left(\vec{x} ; \vec{x}_{1}, \ldots, \vec{x}_{N} ; i \tilde{H}_{R} ; \theta\right)\right)(36)
\end{aligned}
$$

This form of path integration reminds us of the following formula of the statistical mean of an arbitrary physical quantity " $f$ " under the Boltzmann distribution at temperature $T$.

$$
<f>=\frac{\sum_{f^{\prime}} f^{\prime} e^{-\beta E_{f^{\prime}}}}{\sum_{f^{\prime}} e^{-\beta E_{f^{\prime \prime}}}}
$$

In this equation (37) $\beta=\frac{1}{k_{B} T}$ with $k_{B}$, the Boltzmann constant, $T$ is the temperature in the Kelvin unit and $E_{f^{\prime}}$ is the energy of the state $f^{\prime}$. Apart from the normalization factor $\sum_{f}, e^{-\beta E_{f}}$, Eq.(36) is nothing but the formula of the statistical mean (37). with the temperature $T$ as the ratio $\frac{\tilde{H}_{R}}{\Delta t}$.

\subsection{Monte Carlo method}

Once we arrive at the well-known formula in statisitical physics, methods of Monte Carlo integration will be one of familiar tools to calculate the mean path or the path obeying canonical equations of motion. After characterizing the path $\pi$ as a collection of $N$ points, $\pi=\left\{\vec{x}_{k} \mid k=1,2, \ldots, N\right\}$, and starting with an arbitrary path, we can divide our algorithm into the following two successive processes ${ }^{4}$,

(1) examining that the stochastic process achieves stationary state,

(2) calculating the mean value.

In both processes the so called Metropolis algorithm ${ }^{5)}$ is used. Starting with a path $\pi_{0}$ that is initially guessed successive paths are chosen a step at time such that they differ at most in the coordinate of one node as shown in Fig. 2, say $x_{k, l} \rightarrow x_{k, l}^{\prime}=x_{k, l}+\delta x_{l}$ (deformation constant). This step is chosen with a probability $W_{k k^{\prime}}=$ $\min \left(1, \frac{\rho\left(\pi^{\prime}\right)}{\rho(\pi)}\right)$, where 


$$
\rho(\pi)=e^{-\frac{\Delta t}{\bar{H}_{R}} E(\pi)}
$$

is called the Boltzmann factor. In other words, if $\Delta E \equiv$ $E\left(\pi^{\prime}\right)-E(\pi)<0$ the coordinate is altered to $x_{k, l}^{\prime}$ and otherwise it is altered if $e^{-\frac{\Delta l}{\hat{H}_{R}} \Delta E}>\xi$, where $\xi$ is a random variate from the uniform distribution on $(0,1)$.

In the first process we take a sufficient number of steps to erase the influence of the path $\pi_{0}$, initially guessed. To visualize this process, we utilize a coefficient function

$$
R_{F}\left(\vec{x}_{N}\right) \prod_{k=1}^{N} \frac{1}{g_{2}\left(\vec{x}_{k}^{(\theta)}\right)}
$$

that is multiplied to the delta function in Eq.(34). We use the above coefficient function to monitor that the deformation process approaches the stationary state. After this first process the paths so selected are distributed according to Eq.(36). In the second process we only calculate the arithmetic mean of the selected paths.

\section{Simulation}

Ideas are illustrated here in an application to a typical nonlinear control system with 1-input and 2-states. We take an "inverted pendulum" represented by Eqs.(25), (26) and (27) where we set $F_{1}=x_{2}$ and

$$
\begin{gathered}
g_{2}=-\frac{\mu_{1} l \cos x_{1}}{D+\mu_{1}^{2} l^{2} \sin ^{2} x_{1}} \\
F_{2}=\frac{\mu_{1} g l\left(\mu_{1}+\mu_{2}\right) \sin x_{1}}{D+\mu_{1}^{2} l^{2} \sin ^{2} x_{1}}-\frac{\mu_{1}^{2} l^{2} \cos x_{1} \sin x_{1}}{D+\mu_{1}^{2} l^{2} \sin ^{2} x_{1}}\left(x_{2}\right)^{2} \\
V_{\text {cost }}(\vec{x})=Q_{c 1} x_{1}^{2}+Q_{c 2} x_{2}^{2}
\end{gathered}
$$

In the above, $\mu_{1}$ and $\mu_{2}$ are the pendulmum mass and the cart mass, respectively, $l$ is the pendulum length and $D=J\left(\mu_{1}+\mu_{2}\right)+\mu_{1} \mu_{2} l^{2}$ with $J=\frac{\mu_{1} l^{2}}{3}$ is the moment of inertia. The terminal cost is at least 1-time differentiable and we set a complicated form.

$$
\Phi(\vec{x})=Q_{F} e^{-\frac{1}{Q_{F 1}\left(r_{1}-r_{c 1}\right)^{2}+Q_{F 2}\left(r_{2}-r_{c 2}\right)^{2}+Q_{F}}}
$$

Corresponding to this phase function of the final wave function (10), we set the absolute value function as, $R_{F}(\vec{x})=\exp \left(-\left(\frac{x_{1}^{2}}{\sigma_{1}^{2}}+\frac{x_{2}^{2}}{\sigma_{2}^{2}}\right)\right)$. To calculate the wave function we need the function $f$, Eq.(34) and $E$, Eq.(35). The coefficient function $g_{2}(\vec{x})$ above is a function only of $x_{1}$ with no dependence on $x_{2}$. We have calculated simply $\mathcal{F}_{1}=F_{1}, \mathcal{F}_{2}=F_{2}$ and the following.

$$
\mathcal{V}_{\text {cost }}\left(\vec{x} ; H_{R} ; \theta\right)=V_{\text {cost }}-\theta i H_{R} \frac{\partial F_{2}}{\partial x_{2}}
$$

The monitor function is given by Eq.(39). In applying the path deformation process, we must make the generated path $\pi^{\prime}$ avoid points of $g_{2}(\vec{x})=0$. This is easily
Table 1 Conditions and results of calculations

\begin{tabular}{l|c|l|l|l|l}
\hline parameter & $\tilde{H}_{R}$ & 0.05 & 0.005 & 0.0005 & 0.00005 \\
\hline condition & $\delta x_{2}$ & 0.13 & 0.045 & 0.015 & 0.005 \\
\hline \multirow{4}{*}{ results } & $\mathrm{AD} 1[\%]$ & 34.2 & 34.2 & 32.9 & 32.6 \\
\cline { 2 - 6 } & $\mathrm{AD} 2[\%]$ & 36.8 & 34.0 & 32.4 & 30.8 \\
\cline { 2 - 6 } & $\varepsilon\left[\times 10^{-2}\right]$ & 0.140 & 0.106 & 0.100 & 0.099 \\
\hline
\end{tabular}

done by posing the rule that we never accept the paths containing points satisfying $g_{2}(\vec{x})=0$.

System parameters are taken as $\mu_{1}=0.1, \mu_{2}=1, l=1$ and control parameters including parameters of the terminal condition are given as $m=Q_{c 1}=Q_{c 2}=Q_{F}=$ $Q_{F 1}=Q_{F 2}=Q_{F 0}=1$ and $x_{c 1}=x_{c 2}=1$. The corresponding absolute value function is defined by using the parameters $\sigma_{1}=\sigma_{2}=1$. Simulation is done with the parameters $t_{I}=0, t_{F}=10$ and an initial condition of $x_{1}=1.5$ and $x_{2}=0$. Any path is divided into $N=100$ pieces and the maximum cycles of deformation is set as $M=500000^{(3)}$. We set the characteristic constant as $\tilde{H}_{R}=0.05,0.005,0.0005$ and 0.00005 . For each control constant $\tilde{H}_{R}$, as shown in Table 1 we set the deformation constant $\delta x_{2}$ such that accepted deformations are around one over three of total deformation cycles ${ }^{4)}$. In Table 1 , $A D 1[\%]$ and $A D 2[\%]$ are the ratio of the accepted cycles to $M$ in the 1 st process and that in the 2 nd process, respectively. We see trends of the common logarithm values of the monitor function in Fig. 3, where the left part shows the trend in the 1st process while the right part gives that in the second process after achievement to the stationary state. The path $\pi$ or the time development of the state variable $\vec{x}$ is calculated as in Fig. 4 , which is utilized to calculate the control variable $u$ as shown in Fig. 5 according to Eq.(26). To emphasize in these Figs. 4 and 5 fluctuations in the state variable $\vec{x}$ and the control variable $u$, we set the range as $|x| \leq 0.01$ and $|u| \leq 0.2$, respectively. The optimal path must satisfy the canonical equations of motion and the final condition. The canonical equation of motion of the state variable $\vec{x}$ is expressed

(3) For three values of $M$ we calculated errors $\varepsilon$ defined below. The results with $\tilde{H}_{R}=0.00005$ were $\varepsilon=0.105 \times 10^{-2}$, $0.102 \times 10^{-2}$ and $0.099 \times 10^{-2}$ for $M=50000,100000$ and 500000 , respectively. Similar results were obtained for other values of $\tilde{H}_{R}$. We set $M=500000$ expecting that the value $M=500000$ is large enough to lead to sufficiently small error $\varepsilon$. For another parameter $N$, we must take this correspondingly to the value $\tilde{H}_{R}$, because there is a relation $T=\frac{\tilde{H}_{R}}{\Delta t}$ However, we simply take the same value $N=100$ for various $\tilde{H}_{R}$ because in these calculations we mainly examine a relation between the error $\varepsilon$ and $\tilde{H}_{R}$ and the purpose of the report is a proposal of a new algorithm. An examination on how to set these values $\tilde{H}_{R}, M$ and $N$ simultaneously will be reported in forthcoming papers. 


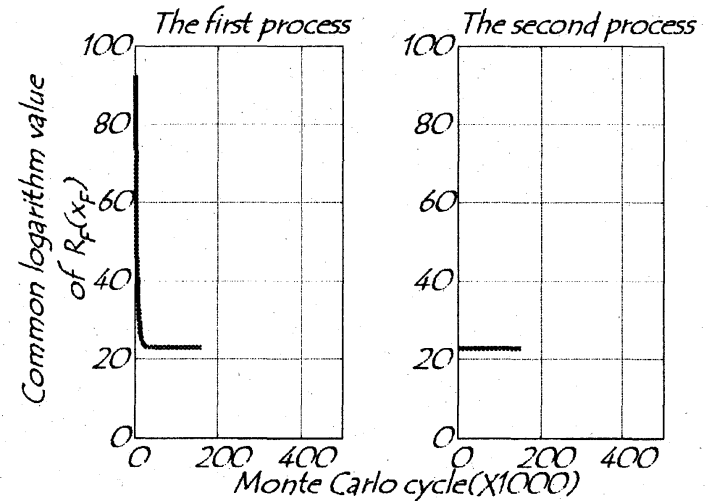

Fig. 3 Trends of the value of the monitor function for $\tilde{H}_{R}=$ 0.00005

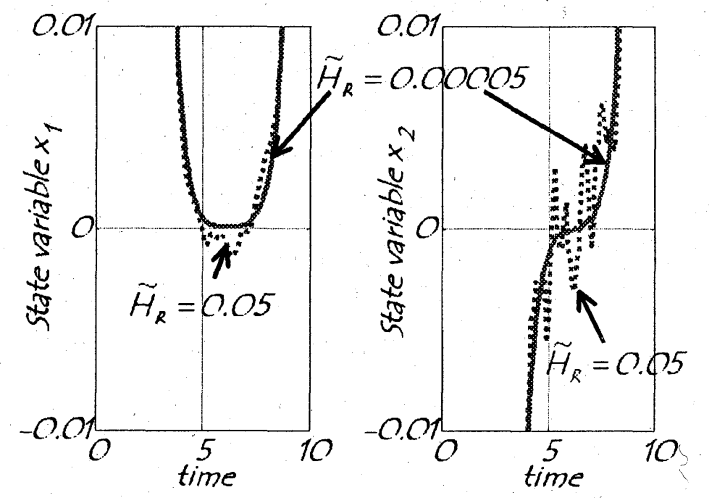

Fig. 4 Simulaiton results of state variables $x_{1}$ and $x_{2}$ in the region $|x| \leq 0.01$ for $\tilde{H}_{R}=0.05$ and 0.00005
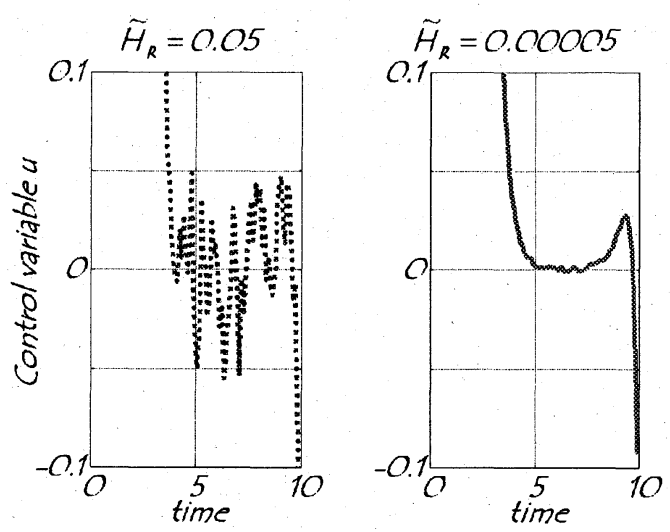

Fig. 5 Simulation results of control variable $u$ in the region $|u| \leq 0.1$ for $\tilde{H}_{R}=0.05$ and 0.00005

as Eq.(B. 5) by setting $H_{R}=0$, which is nothing but the set of state equations (25) and (26). The state equation (25) is automatically satisfied. This is because any path $\pi$ is constrained by the delta function in Eq.(34) where $\mathcal{F}_{1} \rightarrow F_{1}$ in $H_{R} \rightarrow 0$. The state equation (26) also holds identically because we have calculated the control variable $u$ so as to satisfy this state equation. The canonical equations of motion of the canonical variable $\vec{p}_{x}$ and the final condition are Eqs.(B.4) with $H_{R}=0$ and (B.6), respectively. The 1st components of these equations for $p_{x, 1}$ hold because these are given conditions as shown in Appendix C. We must then check whether the calculated path $\pi$ satisfy the 2nd components of Eqs.(B. 4) and (B.6). In a finite difference form, Eqs.(B.4) and (B.6) correspond to Eqs.(C. 11) and (C. 7), respectively, as calculated in Appendix C. Errors of the optimal path $\varepsilon$ are therefore estimated by residuals of the sum of the r.h.s. of Eq.(C. 11) with $H_{R}=0$ and that of Eq.(C. 7) normalized by the average value of $\frac{p_{x, 2}}{\Delta t}$. These sums are divided by the number of the nodes $N=100$ to evaluate the error $\varepsilon$ per one node, which are calculated as in Table 1. We can see from these results that the errors can be reduced by taking smaller value of $\tilde{H}_{R}$.

\section{Summary and Disccusion}

We showed that a probabilistic integration scheme applied to path integral representation of affine nonlinear optimal control systems is useful to calculate two point boundary value problems, or open-loop control problems. After a formulation of nonlinear control systems as a collection of paths in state-space, we transformed the new designer's constant $H_{R}$ to a pure imaginary number, $H_{R}=i \tilde{H}_{R}$. The path integral was then identified with the statistical mean under the Boltzmann distribution, with a real positive constant $\tilde{H}_{R}$ as playing the role of temperature. We thus arrived at the scheme of calculating the path that satisfies two point boundary values as the statistical mean at low "temperature". Ideas were illustrated in a typical nonlinear control system, an inverted pendulum, with 1-input and 2-states. Errors of the canonical equations of motion were calculated to obtain smaller values for smaller value of $\tilde{H}_{R}$. Open-loop calculations are reduced in the report to evaluating well-known formulas of the Boltzmann distribution. We can thus fully make use of various tools of statistical mechanics developed so far. The formulas obtained in the report will appropriately be extended to allow arbitrary time $t$ and location $\vec{x}$ to be utilized in calculations of closed-loop control. These points will be examined in forthcoming papers.

The author would like to thank Dr./Prof. Fujisaki,Y. and Dr./Prof. Oishi, Y. for introducing him information on recent development of random algorithms in nonlinear control theories. He would also like to thank Dr./Prof. Ohtsuka,T. for discussing theories and algorithms on two point boundary value problems. Also comments from the referees are appreciated.

\section{References}

1) U.M.Ascher, R.M.M.Mattheij and R.D.Russell: Numerical Solution of Boundary Value Problems for Ordinary Differ- 
ential Equations, Classics in Applied Mathematics, 13, Society for Industrial and Applied Mathematics (1995)

2) T.Itami: Quantum Mechanical Theory of Nonlinear Control; Proc. of 5th IFAC Symposium Nonlinear Control Systems (NOLCOS'01), 1493/1498(2001)

3) B.Gaveau, T.Jacobson, M.Kac and L.S.Schulman: Relativistic Extension of the Analogy between Quantum Mechanics and Brownian Motion; Physical Review Letters, 53-5, 419/422(1984)

4) H.Gould and J.Tobochnik: An Introduction to Computer Simulation Methods, Addison-Wesley(1996)

5) P.K.MacKeown: Evaluation of Feynman path integrals by Monte Carlo methods; American Journal of Physics, 53-9, 880/885(1985)

6) B.T.Polyak: Random algorithms for solving convex inequalityies; In D.Butnariu, Y.Censor and S.Reich, Inherently Parallel Algorithms in Feasibility and Optimization and their Applications, 409/422(2001)

7) L. I. Schiff:Quantum Mechanics 3rd Ed., McGrawHill(1968)

\section{Appendix A. A c-number Hamiltonian}

We express the Hamiltonian operator $\hat{H}$ in terms of a function $H^{[\theta]}\left(\vec{p}_{x}, \vec{x} ; H_{R}\right)$ which is not an operator and is characterized by a parameter $\theta$. Arguments of the function $H^{[\theta]}$ are the state variable $\vec{x}$, the canonical momentum $\vec{p}_{x}$ and the designer's constant $H_{R}$. In the function $H^{[\theta]}$, we take into account that the state variable $\vec{x}$, the control variable operator $\hat{\vec{u}}$ and the costate variable operator $\hat{\vec{\lambda}}$ do not commute each other, $x_{i} \hat{u}_{\alpha} \neq \hat{u}_{\alpha} x_{i}$, $x_{i} \hat{\lambda}_{j} \neq \hat{\lambda}_{j} x_{i}$ and $\hat{u}_{\alpha} \hat{\lambda}_{i} \neq \hat{\lambda}_{i} \hat{u}_{\alpha}$ and in general $\hat{u}_{\alpha} \hat{u}_{\beta} \neq$ $\hat{u}_{\beta} \hat{u}_{\alpha}$. In this section we not only define the function $H^{[\theta]}$ but also write down the explicit formula for affine systems. We show that the c-number Hamiltonian $H^{[\theta]}$ for affine systems reduces to the conventional Hamiltonian $H \equiv-L-\overleftarrow{\lambda} \cdot \vec{f}$ in $H_{R} \rightarrow 0$

It is convenient to use the bra-ket formulation ${ }^{7)}$ invented by Dirac. The quantum mechanical state is a superposable vector, "ket", |\rangle . The dual is a "bra", $\left\langle\right.$. The wave function $\psi\left(\vec{x}, t ; H_{R}\right)$ is the projection of the ket $|t\rangle$ to the eigen ket $|\vec{x}\rangle$. with the eigenvalue $\vec{x}$, $\psi\left(\vec{x}, t ; H_{R}\right)=\langle\vec{x} \mid t\rangle$. To the eigen ket $\left|\vec{p}_{x}\right\rangle$ corresponds the following formula.

$$
\left\langle\vec{x} \mid \vec{p}_{x}\right\rangle=\frac{1}{\sqrt{\left(2 \pi H_{R}\right)^{n}}} e^{\frac{i}{H_{R}}} \overleftarrow{x} \cdot \vec{p}_{x}
$$

Using the closure properties, $1=\int d \vec{x}|\vec{x}\rangle\langle\vec{x}|$ and $1=$ $\int d \vec{p}_{x}\left|\vec{p}_{x}\right\rangle\left\langle\vec{p}_{x}\right|$, we express the Hamiltonian operator as a superposition of $H^{[\theta]}$. First, we have the following.

$$
\begin{aligned}
\hat{H}= & \int d \vec{x}_{a}\left|\vec{x}_{a}\right\rangle\left\langle\vec{x}_{a}\left|\int d \vec{p}_{x_{a}}\right| \vec{p}_{x_{a}}\right\rangle\left\langle\vec{p}_{x_{a}}\right| \\
& \hat{H} \int d \vec{p}_{x_{b}}\left|\vec{p}_{x_{b}}\right\rangle\left\langle\vec{p}_{x_{b}}\left|\int d \vec{x}_{b}\right| \vec{x}_{b}\right\rangle\left\langle\vec{x}_{b}\right|
\end{aligned}
$$

where Eq.(A.1) and its complex conjugate are used to have

$$
\begin{aligned}
& \hat{H}=\int d \vec{x}_{a} \int d \vec{p}_{x_{a}} \int d \vec{x}_{b} \int d \vec{p}_{x_{b}}
\end{aligned}
$$

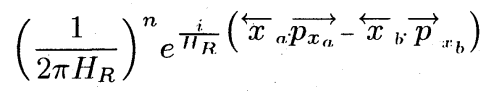

$$
\begin{aligned}
& \left|\vec{x}_{a}\right\rangle\left\langle\vec{p}_{x_{a}}|\hat{H}| \vec{p}_{x_{b}}\right\rangle\left\langle\vec{x}_{b}\right|
\end{aligned}
$$

Next, after we introduce a real parameter $\theta$, we do a variable transformation, $\vec{p}_{x_{a}}=\vec{p}_{x}+\left(\frac{1}{2}-\theta\right) \vec{z}, \vec{x}_{a}=\vec{x}+\left(\frac{1}{2}+\theta\right) \vec{w}$, $\vec{p}_{x_{b}}=\vec{p}_{x}-\left(\frac{1}{2}+\theta\right) \vec{z}$ and $\vec{x}_{b}=\vec{x}-\left(\frac{1}{2}-\theta\right) \vec{w}$, which leads to the equality, $\overleftarrow{x}_{a} \cdot \vec{p}_{x_{a}}-\overleftarrow{x}_{b} \cdot \vec{p}_{x_{b}}=\overleftarrow{x} \cdot \vec{z}+\overleftarrow{w} \cdot \vec{p}_{x}$ Finally, the Hamiltonian operator is given as follows.

$$
\begin{aligned}
& \hat{H}=\iiint \frac{d \vec{w} d \vec{x} d \vec{p}_{x}}{\left(2 \pi H_{R}\right)^{n}} e^{\frac{i}{\Pi_{R}}} \overleftarrow{w} \cdot \vec{p}_{x} \\
& \left|\vec{x}+\left(\frac{1}{2}+\theta\right) \vec{w}\right\rangle\left\langle\vec{x}-\left(\frac{1}{2}-\theta\right) \vec{w}\right| H^{[\theta]}\left(\vec{p}_{x}, \vec{x} ; H_{R}\right)(\mathrm{A} .4)
\end{aligned}
$$

In this formula, the following c-number function

$$
\begin{aligned}
& H^{[\theta]}\left(\vec{p}_{x}, \vec{x} ; H_{R}\right) \equiv \int d \vec{z} e^{\frac{i}{H_{R}} \overleftarrow{x} \cdot \vec{z}} \\
& \left\langle\left\langle\vec{p}_{x}+\left(\frac{1}{2}-\theta\right) \vec{z}|H(\vec{x}, \hat{\vec{u}}, \hat{\vec{\lambda}})| \vec{p}_{x}-\left(\frac{1}{2}+\theta\right) \vec{z}\right\rangle\right.
\end{aligned}
$$

takes an effect of the operator ordering into consideration. Using the above formula of $H^{[\theta]}$, we can calculate the quantity $\hat{H} \delta\left(\vec{x}_{k-1}-\vec{x}_{k}\right)$ appearing in Eq.(18). Partial derivative operation in $\hat{H}$ acts on $\vec{x}_{k-1}, \hat{H} \delta\left(\vec{x}_{k-1}-\vec{x}_{k}\right)=$ $\left\langle\vec{x}_{k-1}|\hat{H}| \vec{x}_{k}\right\rangle$. Using the formula (A.4), since $\left\langle\vec{x}^{\prime} \mid \vec{x}^{\prime \prime}\right\rangle=$ $\delta\left(\vec{x}^{\prime}-\vec{x}^{\prime \prime}\right)$ holds, we obtain that

$$
\begin{array}{r}
\left\langle\vec{x}_{k-1}|\hat{H}| \vec{x}_{k}\right\rangle=\iiint \frac{d \vec{w} d \vec{x} d \vec{p}_{x}}{\left(2 \pi H_{R}\right)^{n}} e^{\frac{i}{H_{R}}} \overleftrightarrow{w} \cdot \vec{p}_{x} \\
\delta\left(\vec{x}_{k-1}-\vec{x}-\left(\frac{1}{2}+\theta\right) \vec{w}\right) \delta\left(\vec{x}-\left(\frac{1}{2}-\theta\right) \vec{w}-\vec{x}_{k}\right) \\
H^{[\theta]}\left(\vec{p}_{x}, \vec{x} ; H_{R}\right)
\end{array}
$$

Carrying out the integration $\int d \vec{x}$ and $\int d \vec{w}$, and rewriting the integral variable $\vec{p}_{x} \rightarrow \vec{p}_{x_{k}}$ lead to the following

$$
\begin{aligned}
\hat{H} \delta\left(\vec{x}_{k-1}-\vec{x}_{k}\right)=\int \frac{d \vec{p}_{x_{k}}}{\left(2 \pi H_{R}\right)^{n}} \\
\quad e^{\frac{i}{\| t_{R}}\left(\overleftarrow{x}_{k-1}-\overleftarrow{x}_{k}\right) \vec{p}_{x_{k} H^{[\theta]}}\left(\vec{p}_{x_{k}}, \vec{x}_{k}^{(\theta)} ; H_{R}\right)}
\end{aligned}
$$

which is nothing but Eq.(19).

For affine systems defined by Eqs.(23) and (24), explicit calculations of the c-number Hamiltonian are given in the following. The control variable $u_{\alpha}$ and the costate variable $\lambda_{i}$ have the following operator representations.

$$
\begin{aligned}
& \hat{u}_{\alpha}=-\left(\bar{R}^{-1}\right)_{\alpha, \underline{\beta}} i H_{R} \nabla^{g_{j}, \underline{\beta}} \underline{\underline{j}} \\
& \hat{\lambda}_{i}=i H_{R} \frac{\partial}{\partial x_{i}}
\end{aligned}
$$

where $\bar{R} \equiv R+{ }^{t} R$ and $\nabla_{i}^{h} \equiv h \frac{\partial}{\partial x_{i}}+\frac{1}{2} \frac{\partial h}{\partial x_{i}}$. The Hamiltonian operator is thereby given by the following formula,

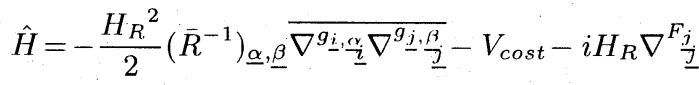


where a symmetrization $\overline{\hat{\omega} \hat{\sigma}} \equiv \frac{\hat{\omega} \hat{\sigma}+\hat{\sigma} \hat{\omega}}{2}$ for arbitrary operators $\hat{\omega}$ and $\hat{\sigma}$ is applied. We can then explicitly calculate the function $H^{[\theta]}$. For arbitrary operator $\hat{O}$,

$$
\begin{aligned}
& O^{[\theta]}\left(\vec{p}_{x}, \vec{x}\right) \equiv \\
& \quad \int d \vec{z}\left\langle\vec{p}_{x}+\left(\frac{1}{2}-\theta\right) \vec{z}|\hat{O}| \vec{p}_{x}-\left(\frac{1}{2}+\theta\right) \vec{z}\right\rangle e^{\frac{i}{H_{R}}} \overleftarrow{x} \cdot \vec{z}
\end{aligned}
$$

is defined. Then the following formulae hold.

$$
\begin{aligned}
\left(h(\hat{\vec{x}}) \hat{p}_{x, i} \hat{p}_{x, j}\right)^{[\theta]} & =p_{x, i} p_{x, j} h \\
& +\left(\frac{1}{2}+\theta\right) i H_{R}\left(p_{x, i} \frac{\partial}{\partial x_{j}}+p_{x, j} \frac{\partial}{\partial x_{i}}\right) h \\
& -\left(\frac{1}{2}+\theta\right)^{2} H_{R}^{2} \frac{\partial^{2} h}{\partial x_{i} \partial x_{j}} \quad \text { (A. 12) } \\
\left(h(\hat{\vec{x}}) \hat{p}_{x, i}\right)^{[\theta]}=p_{x, i} h+\left(\frac{1}{2}+\theta\right) i H_{R} \frac{\partial h}{\partial x_{i}} & \text { (A. 13) }
\end{aligned}
$$

Making use of these formulas, we arrive at the following,

$$
\begin{array}{r}
H^{[\theta]}\left(\vec{p}_{x}, \vec{x} ; H_{R}\right)=\frac{1}{2}\left(\bar{R}^{-1}\right)_{\underline{\alpha}, \underline{\beta}} g_{\underline{i}, \underline{\alpha}} g_{\underline{j}, \underline{\beta}} p_{x, \underline{i}} p_{x, \underline{j}} \\
-\mathcal{V}_{\text {cost }}\left(\vec{x} ; H_{R} ; \theta\right)+p_{x, \underline{i}} \mathcal{F}_{\underline{i}}\left(\vec{x} ; H_{R} ; \theta\right)
\end{array}
$$

where

$$
\begin{array}{r}
\mathcal{V}_{\text {cost }}\left(\vec{x} ; H_{R} ; \theta\right)=V_{\text {cost }}-\theta i H_{R} \frac{\partial F_{\underline{j}}}{\partial x_{\underline{j}}} \\
-\frac{H_{R}^{2}}{2}\left(\bar{R}^{-1}\right)_{\underline{\alpha}, \underline{\beta}}\left\{-\theta^{2}\left(g_{\underline{i}, \underline{\alpha}} \frac{\partial^{2} g_{\underline{j}, \underline{\beta}}}{\partial x_{\underline{i}} \partial x_{\underline{j}}}+g_{\underline{j}, \underline{\beta}} \frac{\partial^{2} \underline{g}_{\underline{i}, \underline{\alpha}}}{\partial x_{\underline{j}} \partial x_{\underline{i}}}\right)\right. \\
\left.-\left(\frac{1}{2}+\theta^{2}\right) \frac{\partial g_{\underline{i}, \underline{\alpha}}}{\partial x_{\underline{i}}} \frac{\partial g_{\underline{j}, \underline{\beta}}}{\partial x_{\underline{j}}}+\left(\frac{1}{4}-\theta^{2}\right) \frac{\partial g_{\underline{i}, \underline{\alpha}}}{\partial x_{\underline{j}}} \frac{\partial g_{\underline{j}, \underline{\beta}}}{\partial x_{\underline{i}}}\right\}
\end{array}
$$

$$
\begin{gathered}
\mathcal{F}_{i}\left(\vec{x} ; H_{R} ; \theta\right)=F_{i}+\left(\bar{R}^{-1}\right)_{\underline{\alpha}, \underline{\beta}} \theta i H_{R} \frac{\partial}{\partial x_{\underline{j}}}\left(g_{i, \underline{\alpha}} g_{\underline{j}, \underline{\beta}}\right) \\
i=1,2 .
\end{gathered}
$$

Any term representing noncommutativity couples to $H_{R}$ or $H_{R}^{2}$. We then see in $H_{R} \rightarrow 0$ that $\mathcal{V}_{\text {cost }} \rightarrow V_{\text {cost }}$, $\mathcal{F}_{1} \rightarrow F_{1}, \mathcal{F}_{2} \rightarrow F_{2}$ and $H^{[\theta]} \rightarrow H=-L-\overleftarrow{\lambda} \cdot \vec{f}$

\section{Appendix B. A stationary phase}

To the path integral representation (22) we can apply stationary phase calculations, when the c-number Hamiltonian $H^{[\theta]}$ has no singularity at $H_{R}=0$. The conditions are given as the vanishing partial derivatives of the phase of the exponential function in Eq.(22). These are written down for the integral variables $\vec{x}_{k}, k=1,2, \ldots, N-1$ and $\vec{p}_{x_{k}}, k=1,2, \ldots, N$. For $k=1,2, \ldots, N-1$,

$$
\begin{aligned}
\frac{\vec{p}_{x_{k+1}}-\vec{p}_{x_{k}}}{\Delta t}= & -\left.\left(\frac{1}{2}-\theta\right) \frac{\partial}{\partial \vec{x}} H^{[\theta]}\left(\vec{p}_{x_{k+1}}, \vec{x} ; H_{R}\right)\right|_{\vec{x}=\vec{x}_{k+1}(\theta)} \\
& -\left.\left(\frac{1}{2}+\theta\right) \frac{\partial}{\partial \vec{x}} H^{[\theta]}\left(\vec{p}_{x_{k}}, \vec{x} ; H_{R}\right)\right|_{\vec{x}=\vec{x}_{k}^{(o)}} \text { (B. 1) }
\end{aligned}
$$

holds, for $k=1,2, \ldots, N$,

$$
\frac{\vec{x}_{k}-\vec{x}_{k-1}}{\Delta t}=\frac{\partial}{\partial \vec{p}_{x_{k}}} H^{[\theta]}\left(\vec{p}_{x_{k}}, \vec{x}_{k}^{(\theta)} ; H_{R}\right)
$$

holds, and for $\vec{x}_{N} \equiv \vec{x}_{F}$,

$$
\begin{aligned}
\vec{p}_{x_{N}}= & -\frac{\partial}{\partial \vec{x}_{F}} \Phi\left(\vec{x}_{F}\right) \\
& -\left.\left(\frac{1}{2}+\theta\right) \frac{\partial}{\partial \vec{x}} H^{[\theta]}\left(\vec{p}_{x_{N}}, \vec{x} ; H_{R}\right)\right|_{\vec{x}=\vec{x}_{N}^{(\theta)}} \Delta t
\end{aligned}
$$

holds, respectively. When the function $H^{[\theta]}$ is continuous and at least 1 -times differentiable, we can take $\Delta t \rightarrow 0$ or $N \rightarrow \infty$ condition. Equations (B. 1), (B. 2) and (B. 3 ) then lead to the following.

$$
\begin{aligned}
& \frac{d \vec{p}_{x}}{d t}=-\frac{\partial H^{[\theta]}\left(\vec{p}_{x}, \vec{x} ; H_{R}\right)}{\partial \vec{x}} \\
& \frac{d \vec{x}}{d t}=\frac{\partial H^{[\theta]}\left(\vec{p}_{x}, \vec{x} ; H_{R}\right)}{\partial \vec{p}_{x}} \\
& \vec{p}_{x_{1}}=-\frac{\partial \Phi\left(\vec{x}_{F}\right)}{\partial \vec{x}_{F}}
\end{aligned}
$$

If $H^{[\theta]} \rightarrow H$ for $H_{R} \rightarrow 0$, Eqs.(B. 4) and (B. 5) in the limit $H_{R} \rightarrow 0$ are nothing but the canonical equations of motion driven by the conventional Hamiltonian $H$. Equation(B.6) gives the final condition (4).

\section{Appendix C. Canonical equations of motion}

The stationary phase calculations have led to the canonical equations of motion (B.4) and (B.5) for the canonical coordinates and the canonical momenta, respectively, and the final condition (B.6). While for affine systems we have the path integral formula (33) of the wave function, where all the integral variables are the state variables. Starting with this integal formula, we have obtained the calculational method of the system with two point boundary values. In this appendix we show that from the path integral formula only in terms of the state variable $\vec{x}$, the canonical equation of motion of the canonical momentum $\vec{p}_{x}$ is derived.

We again use the stationary phase approximation to evaluate Eq.(33). The stationary phase is calculated by the partial derivatives of the function $E$, Eq.(35). We here must take into account the delta functions representing the canonical equation of motion of the state variable component $x_{1}$. For this task it is convenient to take $\theta=-\frac{1}{2}$ (this $\theta$ value is applied throughout this section), $\vec{x}_{k}^{(\theta)}=\vec{x}_{k-1}$, for $k=1,2, \ldots, N$, which leads to

$$
x_{k, 1}=x_{k-1,1}+\mathcal{F}_{1}\left(\vec{x}_{k-1} ; H_{R} ; \theta\right) \Delta t .
$$

From these equations we have, for $i=1,2$,

$$
\frac{\partial x_{k, 1}}{\partial x_{k-1, i}}=\delta_{1, i}+\frac{\partial \mathcal{F}_{1}\left(\vec{x}_{k-1} ; H_{R} ; \theta\right)}{\partial x_{k-1, i}} \Delta t
$$

The stationary phase conditions are given for $k=$ $1,2, \ldots, N$ as follows.

$$
\frac{\partial E}{\partial x_{k, 2}}=
$$




$$
\begin{gathered}
\frac{\partial}{\partial x_{k, 2}} \frac{m}{2\left(g_{2}\left(\vec{x}_{k-1}\right)\right)^{2}}\left(\frac{x_{k, 2}-x_{k-1,2}}{\Delta t}-\mathcal{F}_{2}\left(\vec{x}_{k-1} ; H_{R} ; \theta\right)\right)^{2} \\
+\frac{\partial}{\partial x_{k, 2}}\left\{\frac{m}{2\left(g_{2}\left(\vec{x}_{k}\right)\right)^{2}}\left(\frac{x_{k+1,2}-x_{k, 2}}{\Delta t}-\mathcal{F}_{2}\left(\vec{x}_{k} ; H_{R} ; \theta\right)\right)^{2}\right. \\
\left.+\mathcal{V}_{\text {cost }}\left(\vec{x}_{k} ; H_{R} ; \theta\right)\right\} \\
+\frac{\partial}{\partial x_{k, 2}} \sum_{l=k+1}^{N-1}\left\{\frac{m}{2\left(g_{2}\left(\vec{x}_{l}\right)\right)^{2}}\left(\frac{x_{l+1,2}-x_{l, 2}}{\Delta t}-\mathcal{F}_{2}\left(\vec{x}_{l} ; H_{R} ; \theta\right)\right)^{2}\right. \\
\left.+\mathcal{V}_{\text {cost }}\left(\vec{x}_{l} ; H_{R} ; \theta\right)\right\} \\
+\frac{\partial}{\partial x_{k, 2}} \frac{\Phi\left(\vec{x}_{N}\right)}{\Delta t}=0 \quad(\mathrm{C} .3)
\end{gathered}
$$

where the third term on r.h.s. vanishes for $k=N-1$ and $k=N$ and the second term vanishes for $k=N$. When we set, for $k=1,2, \ldots, N$,

$$
Q_{k}=\frac{m}{\left(g_{2}\left(\vec{x}_{k-1}\right)\right)^{2}}\left(\frac{x_{k, 2}-x_{k-1,2}}{\Delta t}-\mathcal{F}_{2}\left(\vec{x}_{k-1} ; H_{R} ; \theta\right)\right)
$$

we have from Eq.(C. 3)

$$
\begin{gathered}
\frac{Q_{k}}{\Delta t}+\left(-\frac{Q_{k+1}}{\Delta t}-\frac{\partial \mathcal{F}_{2}\left(\vec{x}_{k} ; H_{R} ; \theta\right)}{\partial x_{k, 2}} Q_{k+1}\right. \\
\left.-\frac{1}{m} g_{2}\left(\vec{x}_{k}\right) \frac{\partial g_{2}\left(\vec{x}_{k}\right)}{\partial x_{k, 2}} Q_{k+1}^{2}\right)+\frac{\partial \mathcal{V}_{\cos t}\left(\vec{x}_{k} ; H_{R} ; \theta\right)}{\partial x_{k, 2}} \\
+\sum_{l=k+1}^{N-1}\left\{-\frac{\partial \mathcal{F}_{2}\left(\vec{x}_{l} ; H_{R} ; \theta\right)}{\partial x_{l, 1}} Q_{l+1}\right. \\
\left.-\frac{1}{m} g_{2}\left(\vec{x}_{l}\right) \frac{\partial g_{2}\left(\vec{x}_{l}\right)}{\partial x_{l, 1}} Q_{l+1}^{2}+\frac{\partial \mathcal{V}_{\operatorname{cost}}\left(\vec{x}_{l} ; H_{R} ; \theta\right)}{\partial x_{l, 1}}\right\} \frac{\partial x_{l, 1}}{\partial x_{k, 2}} \\
+\frac{1}{\Delta \iota} \frac{\partial \Phi\left(\vec{x}_{N}\right)}{\partial x_{N, 1}} \frac{\partial x_{N, 1}}{\partial x_{k, 2}}=0
\end{gathered}
$$

for $k=1,2, \ldots, N-2$,

$$
\begin{gathered}
\frac{Q_{N-1}}{\Delta t}+\left(-\frac{Q_{N}}{\Delta t}-\frac{\partial \mathcal{F}_{2}\left(\vec{x}_{N-1}\right)}{\partial x_{N-1,2}} Q_{N}\right. \\
\left.-\frac{1}{m} g_{2}\left(\vec{x}_{N-1}\right) \frac{\partial g_{2}\left(\vec{x}_{N-1}\right)}{\partial x_{N-1,2}} Q_{N}^{2}\right)+\frac{\partial \mathcal{V}_{\operatorname{cost}}\left(\vec{x}_{N-1} ; H_{R} ; \theta\right)}{\partial x_{N-1,2}} \\
+\frac{1}{\Delta t} \frac{\partial \Phi\left(\vec{x}_{N}\right)}{\partial x_{N, 1}} \frac{\partial x_{N, 1}}{\partial x_{N-1,2}}=0
\end{gathered}
$$

and

$$
\frac{Q_{N}}{\Delta t}+\frac{1}{\Delta t} \frac{\partial \Phi\left(\vec{x}_{N}\right)}{\partial x_{N, 2}}=0
$$

We note that Eq.(C. 7 ) is nothing but the final condition of $p_{x, 2}$. Let us define variables $P_{k}, k=1,2, \ldots, N$, as a sequence which satisfies the following conditions

$$
P_{N}=-\frac{\partial \Phi\left(\vec{x}_{N}\right)}{\partial x_{N, 1}}
$$

and for $k=1,2, \ldots, N-1$,

$$
\begin{aligned}
\frac{P_{k+1}-P_{k}}{\Delta t}=-\frac{1}{m} g_{2}\left(\vec{x}_{k}\right) & \frac{\partial g_{2}\left(\vec{x}_{k}\right)}{\partial x_{k, 1}} Q_{k+1}^{2} \\
+\frac{\partial \mathcal{V}_{\operatorname{cost}}\left(\vec{x}_{k} ; H_{R} ; \theta\right)}{\partial x_{k, 1}} & -\frac{\partial \mathcal{F}_{1}\left(\vec{x}_{k} ; H_{R} ; \theta\right)}{\partial x_{k, 1}} P_{k+1} \\
& -\frac{\partial \mathcal{F}_{2}\left(\vec{x}_{k} ; H_{R} ; \theta\right)}{\partial x_{k, 1}} Q_{k+1}
\end{aligned}
$$

We note that Eq.(C. 8) is nothing but the final condition of $p_{x, 1}$. The finite difference formula. (C.9) gives in $H_{R}=0$ the canonical equation of motion of $p_{x, 1}$ in the limit $\Delta t \rightarrow 0$. For these variables $P_{k}$, we can show by mathematical induction the following equalities for $k=0,1,2, \ldots, N-2$.

$$
\begin{gathered}
-\frac{P_{k+1}}{\Delta t}=\sum_{l=k+1}^{N-1}\left\{-\frac{1}{m} g_{2}\left(\vec{x}_{l}\right) \frac{\partial g_{2}\left(\vec{x}_{l}\right)}{\partial x_{l, 1}} Q_{l+1}^{2}\right. \\
\left.-\frac{\partial \mathcal{F}_{2}\left(\vec{x}_{l} ; H_{R} ; \theta\right)}{\partial x_{l, 1}} Q_{l+1}+\frac{\partial \mathcal{V}_{\text {cost }}\left(\vec{x}_{l} ; H_{R} ; \theta\right)}{\partial x_{l, 1}}\right\} \frac{\partial x_{l, 1}}{\partial x_{k+1,1}} \\
+\frac{1}{\Delta t} \frac{\partial \Phi\left(\vec{x}_{N}\right)}{\partial x_{k+1,1}}
\end{gathered}
$$

Using Eqs.(C. 10), (C. 1) and (C. 2) in evaluating the summation term in Eq.(C. 5), we obtain for $k=1,2, \ldots, N-1$ the following equation.

$$
\begin{gathered}
\frac{Q_{k+1}-Q_{k}}{\Delta t}=-\frac{1}{m} g_{2}\left(\vec{x}_{k}\right) \frac{\partial g_{2}\left(\vec{x}_{k}\right)}{\partial x_{k, 2}} Q_{k+1}^{2} \\
+\frac{\partial \mathcal{V}_{\text {cost }}\left(\vec{x}_{k} ; H_{R} ; \theta\right)}{\partial x_{k, 2}}-\frac{\partial \mathcal{F}_{1}\left(\vec{x}_{k} ; H_{R} ; \theta\right)}{\partial x_{k, 2}} P_{k+1} \\
-\frac{\partial \mathcal{F}_{2}\left(\vec{x}_{k} ; H_{R} ; \theta\right)}{\partial x_{k, 2}} Q_{k+1}
\end{gathered}
$$

which leads in $H_{R}=0$ to the canonical equation of motion of $p_{x, 2}$.

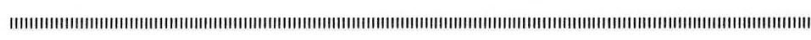

\section{Teturo Iтамі (Member)}

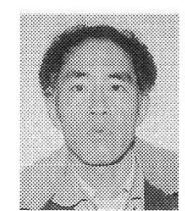

ITAMI Teturo is currently a senior research engineer in Kure Research Laboratory, Babcock-Hitachi K.K.. His research interests include feedback synthesis of nonlinear control and an interplay between control and physics. He is a member of Institute of Systems, Control and Information Engineers and Japan Physical Society.

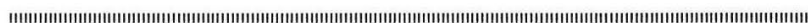

